\title{
Video-assisted thoracoscopic esophagectomy: keynote lecture
}

\author{
Miguel A. Cuesta ${ }^{1}$ Nicole van der Wielen ${ }^{1} \cdot$ Jennifer Straatman $^{1} \cdot$ \\ Donald L. van der Peet ${ }^{1}$
}

Received: 19 February 2016/Accepted: 6 April 2016/Published online: 29 April 2016

(c) The Author(s) 2016. This article is published with open access at Springerlink.com

\begin{abstract}
Minimally invasive esophagectomy (MIE) by thoracoscopy after neoadjuvant therapy results in significant short-term advantages such as a lower incidence of pulmonary infections and a better quality of life (QoL) with the same completeness of resection. After 1 year, a better QoL is still observed for MIE in comparison with the open approach, while having the same survival. Seven issues about implementation of MIE for cancer require discussion: (1) choice of the extension of esophageal resection and use of neoadjuvant therapy; (2) reasons to approach the esophageal cancer by MIE; (3) determining the best minimally invasive approach for gastro-esophageal junction cancers; (4) implementation of evidence-based MIE; (5) standardization of the surgical anatomy of the esophagus based on MIE; (6) future lines of research of MIE; and (7) learning process. In the time of imaging-integrated surgery it is clear that the MIE approach should be increasingly implemented in all centers worldwide having an adequate volume of patients and expertise.
\end{abstract}

Keywords Minimally invasive esophagectomy . Thoracoscopy $\cdot$ Prone position · TIME trial

Miguel A. Cuesta

ma.cuesta@vumc.nl

1 Department of Gastrointestinal Surgery, VU University Medical Center, De Boelelaan 1117, ZH 7F020, 1018 HV Amsterdam, The Netherlands

\section{Introduction}

In 1991, Dallemagne introduced the right thoracoscopic approach in lateral position for esophageal cancer with total lung block, thereby mimicking the conventional approach [1]. Initial reports showed a high conversion rate to thoracotomy and a high respiratory morbidity. Searching for reduction of the conversion rate and the respiratory infection rate, Cuschieri et al. designed the thoracoscopic approach in prone decubitus position so that a total collapse of the lung was no longer necessary for dissecting the esophagus and thereby possibly reducing the rate of respiratory infections [2].

After a feasibility period, the minimally invasive esophagectomy (MIE) approach by thoracoscopy in prone or lateral position or by transhiatal approach is being widely implemented and increasingly performed all over the world for patients with resectable esophageal cancer (EC) to reduce postoperative respiratory complications and to enhance the quality of life by avoiding a right thoracotomy and laparotomy [3-5]. Other important, recent developments in esophageal surgery concern the systematic use of neoadjuvant treatment, such as the use of chemotherapy (MAGIC trial scheme) or chemoradiotherapy (CROSS scheme) [6, 7]. Neoadjuvant therapy for stages 2 and 3 significantly increases 5 -year survival of patients with esophageal cancer in both squamous cell cancer (SCC) as well as adenocarcinomas (Adc).

Currently in discussion in the West is the extension of mediastinal lymphadenectomy. Before, in 1994, the ISDE had defined four types of mediastinal lymphadenectomy in treating esophageal cancer (SCC) according to its extension: the standard, the extended, the total mediastinal and the three-field [8]. The advent of imaging-integrated surgery requires a new look at mediastinal lymphadenectomy. 


\section{Statement about minimally invasive esophagectomy (MIE)}

The minimally invasive esophagectomy should entail the same operation as the standard open esophageal resection with the only difference being the approach: thoracoscopy instead of thoracotomy and laparoscopy instead of laparotomy.

Seven issues will be discussed about the implementation of MIE for cancer:

1. Choice of the extension of esophageal resection, and use of neoadjuvant therapy.

2. Reasons to approach the esophageal cancer by MIE.

3. Determining the best minimally invasive approach for gastro-esophageal junction (GEJ) cancers.

4. Implementation of evidence based MIE.

5. Standardization of the surgical anatomy of the esophagus based on MIE.

6. Future lines of research of MIE.

7. Learning process.

\section{The choice of the extension for MIE esophageal resection and use of neoadjuvant therapy}

Based on information gathered in Japan about the frequency of lymph node metastases according to tumor location [9] and the evidence obtained by randomized controlled trials (RCT) such as the HIVEX trial [10], middle and upper esophageal cancers may be approached by a three-stage thoracoscopy with total mediastinal lymphadenectomy (LN) and laparoscopy with cervical anastomosis after neoadjuvant therapy. In cases of lower esophageal and GEJ, Siewert 1 and 2, a three-stage or a two-stage Ivor Lewis operation is performed, by laparoscopy and thoracoscopy with standard LN with intrathoracic anastomosis after neoadjuvant therapy [11]. If there is a suspicion of enlarged lymph nodes by PET CT-scan in the paratracheal area in these distal tumors, mostly Adc, $\mathrm{LN}$ of these areas is also added. In high-risk patients with distal or GEJ cancers, the laparoscopic transhiatal approach is an option after neoadjuvant therapy.

\section{Reasons to approach the esophageal cancer by MIE}

There is less operative trauma and consequently less morbidity. Performing a thoracoscopy avoids a thoracotomy. Possibly there are fewer postoperative pulmonary complications, especially if no complete pulmonary block had to be used, as done in thoracoscopy in prone position.
In laparoscopic transhiatal dissection, the operation is performed under direct vision and probably with less manipulation and retraction of the mediastinum (heart) and therefore less hemodynamic complications. It will add to a better quality of life and perhaps a better survival [12-17].

All surgical approaches used for open esophagectomy have been implemented for MIE. The transhiatal approach, the three-stage esophageal resection, the Ivor Lewis operation, the thoracoscopy in prone position and the esophageal resection facilitated by robot $[1-5,18-21]$.

\section{Determining the best minimally invasive approach for gastro-esophageal junction (GEJ) cancers}

Gastroesophageal junction adenocarcinomas account for $30-40 \%$ of all esophageal cancers in the West. We useaccepting its limitations-the Siewert classification to locate these tumors leading to implications for the type of neoadjuvant therapy but also for the surgical approach. Many oncologists will indicate neoadjuvant chemotherapy for these tumors. Type 1 is located mainly on the side of the esophagus, type 3 on the side of the gastric cardias and type 2 between both. For type 3 we performed a laparoscopic total gastrectomy [11]. For type 2, an MIE Ivor Lewis procedure is the main choice or the laparoscopic total gastrectomy with an esophagogastrostomy using the Orvil $^{\circledR}$ or a linear stapler anastomosis through the transhiatal approach. Some surgeons will indicate a laparoscopic transhiatal esophageal resection with gastric conduit anastomosis in cervical area and in the case of extensive growth of the tumor along the lesser curvature an open esophageal and gastric resection followed by a colon interposition. Finally for the type 1 tumor, a laparoscopic 2 stage Ivor Lewis or a 3-stage MacKeown approach will be the choice.

The Ivor Lewis approach with intrathoracic anastomosis is a perfect operation for many infracarinal esophageal cancers $[22,23]$. Whilst textbook, it is an operation with a high difficulty grade because of the intrathoracic anastomosis. The operation commences with laparoscopy (celiac trunk lymphadenectomy, gastric dissection, creation of a gastric conduit and hiatal dissection) followed by right thoracoscopy (esophageal resection and lymphadenectomy) and intrathoracic anastomosis through thoracoscopy. While there are different types of intrathoracic anastomosis, nonetheless no evidence posits one as graded superior to the other.

In overview, we have the manual anastomosis or an endto-side anastomosis with a conventional circular stapler $(21,25$ or $28 \mathrm{~mm}$ after a pursestring suture on the esophageal stump or a prepared Orvil device ${ }^{\circledR}$ ). Furthermore, the side-to-side anastomosis can be performed using a 
linear stapler, closing the anterior defect by a transversal suture using conventional suture material or the prepared V-Lock ${ }^{\circledR}$ [24]. Finally the robot-assisted anastomosis is increasingly used permitting a manual high anastomosis in the apex of the thorax because of the ergonomy obtained by the robot [25].

In the Netherlands, anastomotic leaks after MIE Ivor Lewis had initially been reported as high as $14 \%$, subsequently reduced to current rates holding between 5 and $10 \%$ with a low mortality of $2.1 \%$. Surgeons must adhere to a proper algorithm for treating these postoperative anastomotic leaks as early as possible, thereby following the maxim that: "Patients who do not progress every day should be studied immediately by CT-scan and endoscopy for assessment of the anastomosis".

\section{Implementation of evidence based MIE}

There is noteworthy implementation of MIE all over the world. In 2015, using the PubMed ${ }^{\circledR}$ we located 748 papers on MIE esophagectomy and 478 for specifically thoracoscopic esophagectomy. There are four meta-analyses and one randomized controlled trial, being the TIME trial, which compared the total MIE by thoracoscopy in prone and laparoscopy versus the total open approach [12, 2630]. The French MIRO hybrid trial comparing laparoscopy and thoracotomy with intrathoracic anastomosis versus open approach has already been presented at congresses but has not yet been published [31].

The three most important large series are: the Hulscher's series with open transthoracic approach in lateral decubitus (114 patients), the Luketich's series published in 2003 with patients operated by thoracoscopy in lateral position (222 patients), and the Palanivelu's series of 130 patients operated in thoracoscopic prone position. Comparing these, we see an overall survival rate at 3 years of 40, 34 and $42 \%$, respectively. Moreover, the comparative rates of pulmonary complications were 57, 20 and $2.3 \%$, respectively; while the comparative rates of median Intensive Care stay were 6 days, 1 day and 1 day, respectively; and a hospital stay of 19,7 and 8 days, respectively [3, 4, 10].

These striking differences called for evidence-based analysis of effectiveness. Therefore, from 2010 to 2012 the TIME trial was performed in our department. This was a multicentre, open-label randomized controlled trial [30] comparing thoracoscopy in prone position plus laparoscopy versus right posterolateral thoracotomy and laparotomy followed by intrathoracic or cervical anastomosis after neoadjuvant therapy. Primary end point of the trial was determining the rates of respiratory infections in the first 2 weeks and in-hospital stay, while the secondary end points were the quality of the specimen and quality of life
(QoL).We analyzed hospital stay, operative data, postoperative data, complication rate, mortality rates and survival rates.

Concerning the primary outcome, a statistical difference in incidence of postoperative pulmonary infections at end of 2 weeks compared to in-hospital stay was 9 versus $29 \%$ and 12 versus $34 \%$, respectively, in favor of the MIE group. Concerning the secondary outcomes, hospital stay was statistically different (11 and 14 days) in favor of the MIE; but also different answers in the QoL questionnaires (the SF-36 physical component), EORTC C30 (global health) and OES 18 (taking and pain) were found at 2 weeks after operation in favor of MIE. Moreover other outcomes such as the total of retrieved lymph nodes, the rate of $\mathrm{R} 0$ resection (98 and $90 \%$ ), and the in-hospital mortality rates (3.4 and $1.8 \%$ ) were not statistically different between the two groups. Other outcomes, such as operative time, were shorter in the open group whereas blood loss and the VAS score were less in MIE group. Importantly, the outcomes of technical complications such as anastomotic leakage and thoracic complications were not different between the groups, whilst the only exception being incidence of vocal cord palsy that showed an initial difference of 2 versus $14 \%$ in favor of the MIE group. Explanation for this outcome is difficult but has to be sought in the leakage of $\mathrm{CO}_{2}$ from the thorax in the cervical area needed to create a better plane for dissection. The rates of reoperations (14 and $10 \%$, respectively) were no different between the two groups. Moreover, at 1-year follow up there were no differences in overall and disease-free survival rates between the two groups (around $75 \%$ ) yet the QoL questionnaires point out some differences at the 1 -year juncture. The global health, the pain and the physical component of the SF-36 were still statistically different after 1 year in favor of the MIE intervention. Explanation for this is obtained by the advantage of avoidance of the thoracotomy with prevention of the postthoracotomy syndrome [32].

Furthermore, there are four different MIE approaches: (a) the lateral thoracoscopic position, the prone position and the semiprone position [1, 2, 33]; (b) the Hybrid MIE type 1 in which a laparoscopy is combined with a right thoracotomy as in the French MIRO trial [31], (c) the Hybrid MIE 2 that combines a thoracoscopy and the laparotomy [13], and (d) the robot-assisted thoracoscopy with standard laparoscopy [21].

Concerning the semiprone position as proposed in Japan, this seems an important addition to the standard prone approach. This includes the possibility to balance the patient from prone to right semi lateral in order to better visualize the supracarinal area and do a better lymphadenectomy along both recurrent laryngeal nerves [33]. Concerning the thoracoscopy in prone approach there are 
some differences in the position of trocars between Japanese and western world surgeons. The first positions the trocars anteriorly of the scapula adding mostly a small thoracotomy for retraction, whereas the second positions the trocars posteriorly, between the scapula and the spine, adding only a small thoracotomy at the end of the procedure for retrieval of the specimen and introduction of the circular stapler in the case of Ivor Lewis operation.

Differences between the lateral and prone position show that, while in the prone position there is no necessity for selective intubation in the case of cervical anastomosis (we use an insufflation of 7-8 $\mathrm{mm} \mathrm{Hg} \mathrm{CO}$ for helping retraction of the lung) in the lateral position selective intubation is usually used. When questions arise whether a quick conversion to thoracotomy is needed because of bleeding, sufficient experience assures that conversion may be performed in both positions.

Differences between prone and lateral position are studied by Kubo et al. with two cohorts of 28 patients in lateral and 30 in prone position. Blood loss and duration of systemic inflammatory response were significantly better in the prone group, with a tendency of the respiratory complications to be also lower in the prone group. Their conclusion was that while thoracoscopy in lateral position was safe and feasible, the prone position might be a potentially less invasive procedure than the lateral position [34].

The FREGAT French group compared the 30-day postoperative mortality (POM) between two important cohorts of patients (663 MIE and 2346 open esophagectomy patients) of the French register. Thirty-day postoperative mortality was 3.3 versus $5.7 \%$, the in-hospital mortality 5.6 versus $8.1 \%$ and at 90-day mortality 6.9 and $10 \%$, respectively; where the 30-day POM was significantly favoring the MIE. This study suggests that POM is significantly reduced after MIE for EC. This is highly valuable evidence for aiding in decision-making regarding an optimal (hybrid $1 \mathrm{MIE}$ ) approach [35].

Concerning long-term survival, Burdall et al. reported three large series in the UK: the open approach (83 patients), the MIE (64 patients) and the hybrid type 1 (187 patients). They found in the long term that the probability of the length of survival for the three groups, even with no adjustment for $\mathrm{T}$ or $\mathrm{N}$ stage, was greatest for the MIE group [36].

\section{Standardization of the surgical anatomy of the esophagus based on MIE}

The information gathered by MIE has permitted us to describe the concept of the meso-esophagus in the subcarinal area of the thoracic esophagus. During thoracoscopy in prone position we have observed that all structures at subcarinal level, vessels, lymph vessels and nerves are coming from the side of the thoracic aorta to the esophagus. There is a double fascia between aorta and the esophagus that has to be divided in order to perform a systematic step-by-step MIE. In the supracarinal esophagus it is different because the vessels, nerves and lymph vessels are coming on both sides of the esophagus. Description of which structures have to be divided and which to be preserved at this level is important for an adequate esophageal resection and lymphadenectomy with preservation of the arterial vascularization of the trachea and bronchi and proper innervation to the lungs [37, 38].

\section{Future lines of research of MIE}

There are some ongoing RCT's such as the ROBOT trial that compares the open esophagectomy with the thoracoscopic approach assisted by the robot [21], but also RCT's that will compare the MIE with the open and hybrid approaches. In the UK and in Japan surgeons still harbor doubts about the advantages of MIE, therefore three new trials have been started. In the UK, the ROMIO trial with three arms: the MIE, the hybrid 1 and the open [39]. In Japan surgeons are comparing the prone position with the open esophagectomy and in China they are going to initiate a RCT comparing the lateral MIE with the open approach [40].

\section{Learning process}

To initiate teaching of the MIE approach, surgeons of a designated proctored Upper GI group will need to have access to an adequate volume of patients with EC and have gained enough experience in open esophagectomy and minimally invasive surgery. Moreover, with the approval of the direction of the hospital and the department they have to organize a dedicated team (at least with 2 surgeons) and visit a center of excellence to learn how this type of intervention has to be performed. Consequently, apprentice learners, will under the guidance of an authorized mentor need to be monitored while carrying out several MIE procedures in their own hospital.

\section{Conclusion}

Minimally invasive esophagectomy after neoadjuvant therapy results in significant advantages on the short term such as lower incidence of pulmonary infections and a better short-term QoL with the same completeness of resection. After 1 year there is still a better QoL with the 
same survival rate. Three more RCT's are planned to follow up on the established practice of video-assisted thoracoscopic esophagectomy; together with the publication of the French MIRO hybrid trial, we may expect significant improvements in reducing morbidity and increasing the benefits of the intrathoracic anastomosis.

In the time of imaging-integrated surgery it is clear that the MIE approach should be increasingly implemented in all centers worldwide having an adequate volume of patients and expertise.

\section{Compliance with ethical standards}

Conflict of interest All authors have no conflict of interest or financial ties to disclose.

Open Access This article is distributed under the terms of the Creative Commons Attribution 4.0 International License (http://crea tivecommons.org/licenses/by/4.0/), which permits unrestricted use, distribution, and reproduction in any medium, provided you give appropriate credit to the original author(s) and the source, provide a link to the Creative Commons license, and indicate if changes were made.

\section{References}

1. Dallemagne B, Weerts JM, Jehaes C. Thoracoscopic esophageal resection. In: Cuesta MA, Nagy AG, editors. Minimally invasive surgery in gastrointestinal cancer. Edingburgh: Churchill Livingstone; 1993. p. 59-68.

2. Cuschieri A. Thoracoscopic subtotal esophagectomy. Endosc Surg Allied Technol. 1994;2:21-5.

3. Luketich JD, Alvelo-Rivera M, Buenaventura PO, et al. Minimally invasive esophagectomy: outcomes in 222 patients. Ann Surg. 2003;238:486-94.

4. Palanivelu C, Prakash A, Senthilkumar R, et al. Minimally invasive esophagectomy: thoracoscopic mobilization of the esophagus and mediastinal lymphadenectomy in prone positionexperience of 130 patients. J Am Coll Surg. 2006;2013:7-16.

5. Scheepers JJ, Veenhof XA, van der Peet DL, et al. Laparoscopic transhiatal resection for malignancies of the distal esophagus: outcome of the first 50 resected patients. Surgery. 2008;143:278-85.

6. Cunningham D, Allum WH, Stenning SP, et al. Perioperative chemotherapy versus surgery alone for resectable gastroesophageal cancer. N Engl J Med. 2006;355:11-20.

7. Van Hagen P, Huslhof MC, van Lanschot JJ, et al. Preoperative chemoradiotherapy for esophageal or junctional cancer. N Engl J Med. 2012;366:2074-84.

8. Bumm R, Wong J. More or less surgery for esophageal cancer: extent of lymphadenectomy for squamous cell carcinoma-how much is necessary ? Dis Esophagus. 1994;7:151-5.

9. Akiyama H, Tsurumaru M, Ono Y, et al. Background of lymph node dissection for squamous cell carcinoma of the esophagus. In: Sato T, Lizukan T, editors. In color atlas of surgical anatomy for esophageal cancer. New York: Springer; 1992. p. 9-24.

10. Hulscher JBF, van Sandick JW, de Boer AGEM, et al. Extended transthoracic resection compared with limited transhiatal resection for adenocarcinoma of the esophagus. N Engl J Med. 2002;347:1662-9.
11. Siewert JR, Stein HJ. Carcinoma of the cardia: carcinoma of the gastroesophageal junction. Classification, pathology and extent of resection. Dis Esophagus. 1996;9:173-82.

12. Biere SSAY, Cuesta MA, Van der Peet DL. Minimally invasive versus open esophagectomy for cancer: a systematic review and meta-analysis. Minerva Chirurgica. 2009;64:121-33.

13. Osugi $H$, Takemura $M$, Higashino $M$, et al. A comparison of video-assisted thoracoscopic oesophagectomy and radical lymph node dissection for squamous cell cancer of the oesophagus with open operation. Br J Surg. 2003;90:108-13.

14. Taguchi S, Osugi H, Hirashino M, et al. Comparison of threefield esophagectomy for esophageal cancer incorporating open or thoracoscopic thoracotomy. Surg Endosc. 2003;17:1445-50.

15. Gemmill EH, McCulloch P. Systematic review of minimally invasive resection for gastro-oesophageal cancer. $\mathrm{Br} \mathrm{J}$ Surg. 2007;94:1461-7.

16. Smithers BM, Gotley DC, Martin I, Thomas JM. Comparison of the outcomes between open and minimally invasive esophagectomy. Ann Surg. 2007;245:232-40.

17. Maas KW, Biere SS, van Hoogstraten IM, et al. Immunological changes after minimally invasive or conventional esophageal resection for cancer: a randomized trial. World J Surg. 2014;38:131-7.

18. Cuesta MA, van der Peet DL, Biere SSAY, Scheepers JJG, Heijnen BHM. Laparoscopic transhiatal esophagectomy. In: Puntambeker SP, Cuesta MA, editors. Atlas of minimally invasive surgery in esophageal carcinoma. New York: Springer; 2010. p. 171-89.

19. Cuesta MA, Scheepers JJG, Oosterhuis W, Biere SSAY, Van der Peet DL, Heijnen BHM. Thoracoscopic esophageal resection for cancer in prone decubitus position: operative technique. In: Puntambeker SP, Cuesta MA, editors. Atlas of minimally invasive surgery in esophageal carcinoma. New York: Springer; 2010. p. 149-69.

20. Cadiere GB, Dapri G, Himpens J, et al. Ivor Lewis esophagectomy with manual esofagogastric anastomosis by thoracoscopic in prone position and laparoscopy. Surg Endosc. 2010;24:1482-5.

21. Van der Sluis PC, Ruurda JP, Verhage RJ, et al. Oncologic longterm results of robotic assisted minimally invasive thoraco-laparoscopic esophagectomy with two field lymphadenectomy for esophageal cancer. Ann Surg Oncol. 2015;22(Suppl 3):1350-6.

22. Lewis I. The surgical treatment of carcinoma of the oesophagus with special reference to a new operation for growths of the middle third. Br J Surg. 1946;34:18-31.

23. Luketich JD, Pennathur A, Awais O, et al. Outcomes after minimally invasive esophagectomy. Review of over 1000 patients. Ann Surg. 1000;2012(256):95-103.

24. Maas KW, Biere SSAY, Scheepers JJG, et al. Minimally invasive intrathoracic anastomosis after Ivor Lewis Esophagectomy for cancer. A review of transoral or transthoracic use of staplers. Surg Endosc. 2012;26:1795-802.

25. del Val Diez I, Loureiro C, McCulloch P. The IDEAL prospective development study format for reporting surgical innovations. An illustrative case study of robotic oesophagectomy. Int J Surg. 2015;19:104-11.

26. Verhage RJ, Hazebroek EJ, Boone J, et al. Minimally invasive surgery compared to open procedures in esophagectomy for cancer: a systematic review of the literature. Minerva Chir. 2009;64:135-46.

27. Sgourakis G, Gockel I, Radtke A, et al. Minimally invasive versus open esophagectomy: meta-analysis of outcomes. Dig Dis Sci. 2010;55:3031-40.

28. Nagpal K, Ahmed K, Vats A, et al. Is minimally invasive surgery beneficial in the management of esophageal cancer ? A metaanalysis. Surg Endosc. 2010;24:1621-9. 
29. Biere SSAY, Maas KW, Cuesta MA, van der Peet DL. Cervical or thoracic anastomosis after esophagectomy for cancer: a systematic review and meta-analysis. Dig Surg. 2011;28:29-35.

30. Biere SSAY, van Berge Henegouwen MI, Maas KW, et al. Minimally invasive versus open esophagectomy for patients with esophageal cancer: a multicentre, open-label, randomised controlled trial. Lancet. 2012;379:1887-92.

31. Briez N, Piessen G, Torres F, et al. Effects of hybrid minimally invasive oesophagectomy on major postoperative pulmonary complications. Br J Surg. 2012;99:1547-53.

32. Maas KW, Cuesta MA, van Berge Henegouwen MI, et al. Quality of life and late complications after minimally invasive compared to open esophagectomy: results of a randomized trial. World $\mathbf{J}$ Surg. 2015;39:1986-93.

33. Kawakubo H, Takeuchi H, Kitagawa Y. Current status and future perspectives on minimally invasive esophagectomy. Korean $\mathbf{J}$ Thorac Cardiovasc Surg. 2013;46:241-8.

34. Kubo N, Ohira M, Yamashita Y, et al. Thoracoscopic esophagectomy in the prone position versus in the lateral position for patients with esophageal cancer: a comparison of short term surgical results. Surg Laparosc Endosc percutan tech. 2014;24:158-63.

35. Messager M, Pasquer A, Duhamel A, et al. Laparoscopic gastric mobilization reduces postoperative mortality after esophageal cancer surgery: a French Nationwide Study. Ann Surg. 2015;262:817-23.

36. Burdall O, Boddy AP, Fullick J, et al. A comparative study of survival after minimally invasive and open oesophagectomy. Surg Endosc. 2015;29:431-7.

37. Cuesta MA, Weijs TJ, Bleys RLAW, et al. A new concept of the anatomy of the thoracic esophagus: the meso-esophagus. Observational study during thoracoscopic esophagectomy. Surg Endosc. 2015;29:2576-82.

38. Weijs TJ, Ruurda JP, Luyer MDP et al. Preserving the pulmonary vagus nerve branches during thoracoscopic esophagectomy. Surg Endosc. 2015 (epub ahead of print).

39. Avery KN, Metcalfe C, Berrisford R, et al. The feasibility of a randomized controlled trial of esophagectomy for esophageal cancer-the romio (randomized oesophagectomy: minimally invasive or open) study: protocol for a randomized controlled trial. Trials. 2014;15:200.

40. Kataoka K, Takeuchi H, Mizusawa J, et al. A randomized Phase III trial of thoracoscopic versus open esophagectomy for thoracic esophageal cancer: Japan Clinical Oncology Group Study JCOG 1409. Jpn J Clin Oncol. 2016;46:174-7. 